\title{
THE DETERMINATION AND SIGNIFICANCE OF THE ELECTRICAL AXIS OF THE HUMAN HEART*
}

\author{
FR.ANCIS R. DIEUAIDE, M.D. $\dagger$
}

BALTIMORE

The subject of the electrical axis of the heart has been receiving more and more attention in recent years. In the first edition of Lewis' work "The Mechanism of the Heart Beat" ${ }^{1}$ it is not mentioned. Considerable attention, however, is given the subject in the second edition. ${ }^{2}$ It is not too much to say that a thorough understanding of the relations of the axis is a necessary foundation of knowledge of apparently simple features of the electrocardiogram. This paper has for its purpose the presentation of a new method of evaluating the electrical axis, and a brief discussion of some aspects of the study of the subject.

\section{DEFINITION}

By the term "electrical axis" is meant the line along which the resultant of the electrical forces acting in the heart at any instant is expressed. Or, from another point of view, it corresponds, as Lewis puts it," to "the average direction in which the excitation wave is tending to move at the corresponding instant of time." This axis then is constantly shifting during the progress of the excitatory process. Therefore, one speaks of "the" electrical axis only in a more restricted sense, meaning an axis at a particular instant or a resultant axis.

\section{TER M I NOLOGY}

The electrical axis was first discussed by Waller, ${ }^{3}$ and this worker has subsequently made many observations in this field. Waller arrived at the idea of the electrical axis by seeking an explanation of the different values of corresponding deflections of the galvanometer string obtained in various leads. His system of leads is relatively complicated and his terminology is obscure. The direction of the electrical axis is

* From the Cardiographic Laboratory of the Johns Hopkins University and Hospital.

+ Working under the tenure of the William Bingham Fellowship in Medicine.

1. Lewis, T.: The Mechanism of the Heart Beat, London, 1911.

2. Lewis, T.: The Mechanism and Graphic Registration of the Heart Beat, London and New York, 1920.

3. Waller, A. D.: Electromotive Properties of the Human Heart, Brit. M. J. 2:751 (Oct. 6) 1888. Electromotive Changes Connected with the Beat of the Mammalian Heart, Phil. Tr. Roy. Soc. London, B 80:169, 1889. 
expressed in terms of trigonometry. Waller worked out empirically, and later ${ }^{4}$ proved geometrically a rather simple formula for the calculation of the angle between the electrical and the vertical axes:

$$
\text { (1) } \operatorname{Tan} a=2 \frac{\mathrm{R}}{\mathrm{R}+\mathrm{L}}
$$

in which " $a$ " is the angle between the electrical and the vertical axis; $\mathrm{R}$ is the value of the deflection derived from the right arm and left foot, and $L$ is the value of that derived from the left arm and left foot. This formula can, of course, be used for Einthoven's angle " $a$ " des-

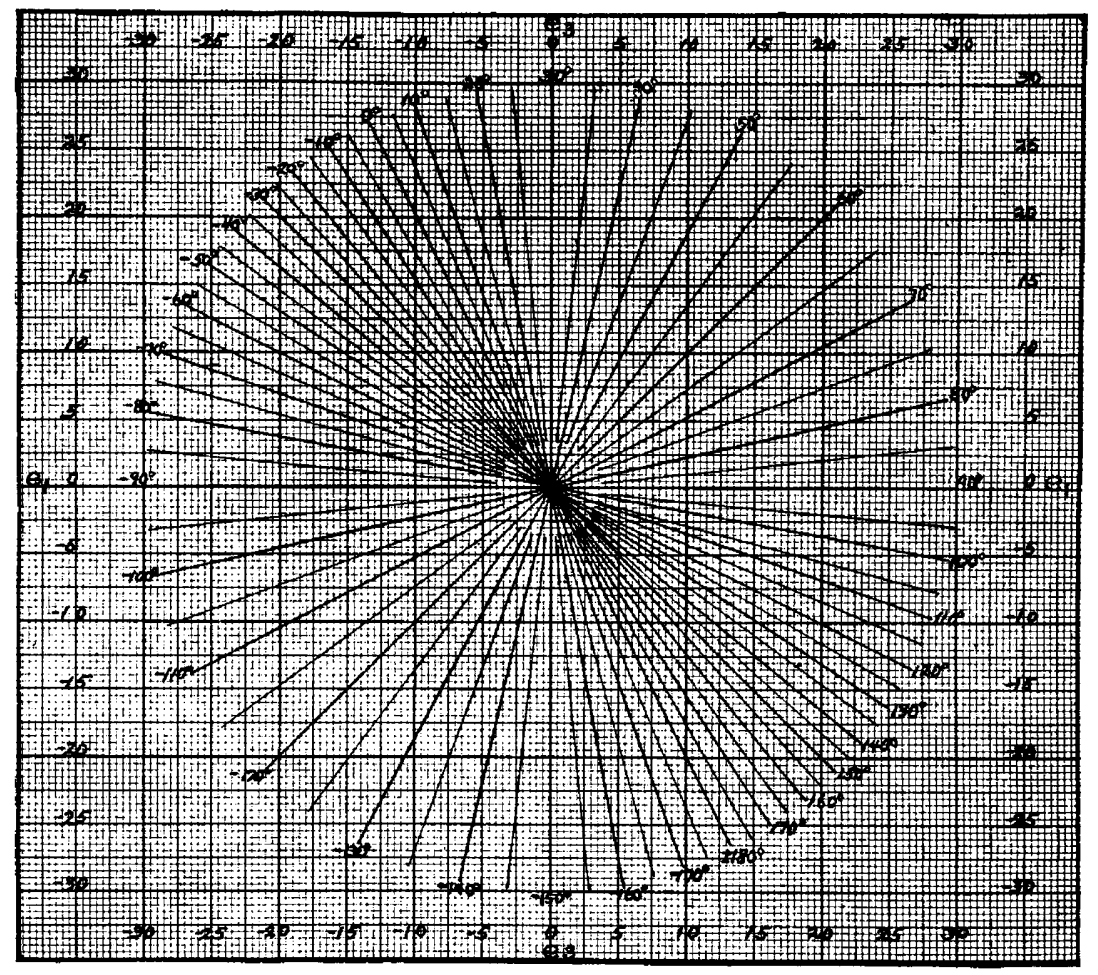

Angle " $a$ " in terms of $\mathcal{c}$ I and $e$ III. For description see text.

cribed below by substituting "cotangent $a$ " for "tangent $a$." Waller's usage has not been followed by writers on the subject.

Our terminology is that of Einthoven. ${ }^{5}$ Selecting from the number of leads available the three in common use, he showed that the values

4. Waller, A. D.: Various Inclinations of Electrical Axis, Pt. 1, Proc. Roy. Soc. London, B 86:507 (July) 1913.

5: Einthoven, W.: Le Télécardiogramme, Arch. Internat. Physiol. 4:132, 1906. Weiteres über das Elektrokardiogramm, Arch. f. d. ges. Physiol. 122: 517 (May) 1908. 
of the deflections so obtained are related as in his equation, according to which at a given instant in the cycle of the heart beat, the value of Lead II is equal to the sum of the values of Leads I and III.

$$
\text { (2) Lead II = Lead I + Lead III }
$$

He later explains this relation by comparison with the movement of a force within an equilateral triangle in which the projections of the course of the force on the sides of the triangle necessarily obey this law. The proof of this is conveniently presented in Mann's recent paper. ${ }^{6}$ A line between the center of the triangle and any point on the course of the moving force would represent the electrical axis at that instant. The values of the deflections, and, hence, of the length of the axis are expressed in tenths of a millivolt. Einthoven refers to the length of the axis as the "manifest" value, but the term "absolute" would be clearer. The axis is defined by the angle, called " $a$," between its plane and the horizontal, which is taken as the zero line. Angles above the horizontal are referred to as negative while those below are said to be positive. This is contrary to our mathematical usage, as pointed out by Carter, ${ }^{7}$ but it would seem best in view of the extensive work of Einthoven, ${ }^{8}$ Lewis, ${ }^{9}$ and Fahr ${ }^{10}$ to continue to use this terminology.

FINDING THE ANGLE

The fundamental equations showing the relations of the leads and the length and direction of the axis as worked out by Einthoven, Fahr and de Waart $^{8}$ are:

(3) $\mathrm{e}_{\mathrm{i}}=\mathrm{E} \cos a$

(4) $\mathrm{e}_{\mathrm{e}}=\mathrm{E} \cos \left(a-60^{\circ}\right)$

(5) $\mathrm{e}_{3}=\mathrm{E} \cos \left(120^{\circ}-a\right)$

In which $e_{1}, e_{2}$ and $e_{3}$ represent the values of the deflections in the three leads respectively; $\mathrm{E}$ is the manifest value or length of the axis and " $a$ "

6. Mann, H.: Method of Analyzing the Electrocardiogram, Arch. Int. Merl. $25: 283$ (March) 1920.

7. Carter, E. P.; Richter, C. P., and Greene, C. H.: Graphic Application of the Principle of the Equilateral Triangle, etc., Johns Hopkins Hosp. Bull. 30:162 (June) 1919.

8. Einthoven, W.; Fahr, G., and de Waart, A.: Ueber die Richtung und die manifeste Grosse, etc., Arch. f. d. ges. Physiol. 150:275 (March) 1913.

9. Lewis, T.: Spread of the Excitatory Process, Phil. Tr. Roy. Soc. Lond. 207: B, 221, 1916.

10. Fahr, G. E.: Analysis of Spread of Excitation Wave, Arch. Int. Med. 25:146 (Feb.) 1920. Fahr, G., and Weber, A.: Ueber die Ortsbestimmung der Erregnung im menschlichen Herzen, Deutsch. Arch. f. klin. Med. 117:361 (June) 1915 . 
the angle between the axis and the horizontal. From these data formulas were evolved the determination of " $a$ " as follows:

$$
\begin{aligned}
& \text { (6) } \operatorname{Tan} a=\frac{2 \mathrm{e}_{2}-\mathrm{e}_{1}}{\mathrm{e}_{1} \sqrt{3}} \\
& \text { (7) } \operatorname{Tan} a=\frac{2 \mathrm{e}_{3}+\mathrm{e}_{1}}{\mathrm{e}_{1} \sqrt{3}} \\
& \text { (8) Tan } a=\frac{\mathrm{e}_{2}+\mathrm{e}_{3}}{\left(\mathrm{e}_{2}-\mathrm{e}_{3}\right) \sqrt{3}}
\end{aligned}
$$

On the basis of these formulas, Einthoven published tables by which the angle " $a$ " may be determined after the values of the deflections are reduced proportionately so that the greatest is equal to 10 . In a later article $^{11}$ is another table based on the same principle differently applied. One or the other of these tables, chiefly the former, has been used in the past by most of those interested in this subject.

Pardee ${ }^{12}$ has shown that it is possible to tell by inspection of the positivity or negativity of the three leads in which quadrant the axis lies. This is because no combination of signs in the three leads occurs in more than one quadrant, which is a direct consequence of the geometric relationship pointed out by Einthoven. ${ }^{8}$

Fahr and Weber ${ }^{10}$ proposed a method of direct geometric construction of the angle. The values of the deflections in two leads are measured off in any units convenient on lines inclined to one another at an angle of 60 degrees. The line representing the electrical axis runs from the vertex of the angle of 60 degrees through the junction of perpendiculars erected at the points measured off. This method is cumbersome and does not lend itself to routine use, especially in the case of angles less than 0 or greater than 90 degrees.

Recently from this laboratory Carter, Richter and Greene, ${ }^{7}$ independently of the previous suggestion, set forth an adaptation of the same principle to the equilateral triangle. In this method a figure is used in which the geometric construction of the lead values commonly met is inserted in an equilateral triangle, about which is circumscribed a concentric circle appropriately divided into degrees. Thus the angle is found by the projection on the circle of a straight line through the center and through the point representing the lead values. This method has been in constant and extensive use in this laboratory since its inception.

In the new edition of the "Mechanism" Lewis " offers for use a figure showing the relative values in the three leads of a given manifest

11. Einthoven, W.: Bergansius, F. L., and Bijtel, J.: Gleichzeitige Registrirung elektrischer Ercheinungen, etc., Arch. f. d. ges. Physiol. 164:167 (June) 1916.

12. Pardee, H. E. B.: Form of the Electrocardiogram. J. A. M. A. 62:1311 (April 25) 1914. 
value, and the changes they undergo as the axis rotates from 0 through 180 degrees to 0 again. This is not of practical value because of the difficulty, amounting almost to impossibility, of proportioning the lead values of widely varying manifest values at unknown angles.

In an interesting variation of the geonetric method, Mann plots the manifest values at successive moments and connects the points by a smooth curve." He uses rectangular co-ordinates of which " $x$ " is given directly by the value of Lead I and " $y$ " must be calculated by a formula from the values of Leads II and III. The result is a "monocardiogram" which is essentially the figure obtained by connecting the ends of the electrical axes as charted by Williams. ${ }^{1: 3}$ It may be plotted directly on the equilateral triangle divided as in Carter's Figure 5, without the necessity of calculating the "y" value.

It should be noted that Mann in his figures reverses the direction of the deflections in all the leads as related to the triangle, and plots positive values of " $x$ " (= Lead I) to the left. This is at variance with the standard method of connecting the lead derivations with the string. That is, in taking the leads the lower end of the string is connected with the derivation which is normally negative, or corresponds to the zinc pole of a Daniell cell. In normal cases, therefore, the current travels down the string which is thereby deflected to the observers's right (provided the polarity of the electromagnet be properly adjusted), the movement being later described as upright. Compare Mann's Figure 2 with Lewis' Figure 13 and Williams' Figure 5.

\section{THE METHOD SUGGESTED}

As Einthoven pointed out, there is a constant ratio between the values of the three leads with a given electrical axis. ${ }^{8}$ It also follows from his formulas, 6,7 and 8 above, that the angle " $a$ " is determined by the ratio between the values of any two leads. This is readily apparent on inspection of the triangle as presented by Carter. But none of the methods suggested for evaluating the angle " $a$ " has taken advantage of this relation, although it is, indeed, the most simple and fundamental relationship between the lead values and the angle " $a$." Table 1 gives the ratios between the values of Leads I and III at the various angles. Such a table is very much more simple to use than those of Einthoven. The ratios are derived by dividing equation (3) by equation (5), thus:

$$
\begin{aligned}
& \text { (9) } \frac{\mathrm{e}_{1}}{\mathrm{e}_{\mathrm{s}}}=\frac{\mathrm{E} \cos a}{\mathrm{E} \cos (120-a)} \\
& \text { (10) } \frac{\cos a}{\cos (120-a)}=\frac{\mathrm{e}_{\mathrm{L}}}{\mathrm{e}_{\mathrm{a}}}=\text { ratio desired. }
\end{aligned}
$$

13. Williams, H. B.: Cause of Phase Difference, etc., Am. J. Physiol. 35: 292 (Oct.) 1914. 
Table 1 is presented graphically in Figure 1, a chart which has been found very easy to use and which gives the result quickly. The ordinates represent values of Lead I, positive above and negative below the horizontal axis. The abscissae represent values of Lead III, positive to the right and negative to the left of the vertical axis. The values of the angles " $a$ " are represented in accordance with Table 1 , at the intersections of the lead values giving the proper ratios. To take an example, suppose the values +9.5 and +16.5 are accepted for Leads I and III of an electrocardiogram. In using the chart, one locates the point representing these values and the angle " $a$ " is given by the relation of that point to the radii drawn in. In this case the point representing $\mathrm{e}_{1}=+9.5$ and $\mathrm{e}_{3}=+16.5$ is approximately at +69 degrees. Also the ratio in this case is $0.57+$ which the table of ratios shows to correspond to between +70 degrees and +65 degrees, much nearer the former. This figure gives the value of the angle " $a$ " with all the accuracy that is desirable.

TABLE 1.-The Angle " $a$ " in Terms of the Ratio, e 1 /e 3 . To Find the ANGle " $a$ " Divide The Value of Lead I by That of Lead III and Under the Proper Signs for the Leads Seek the Angle Opposite the Ratio So OBtained

\begin{tabular}{|c|c|c|c|c|c|c|c|c|}
\hline \multirow{2}{*}{ Rutio } & \multicolumn{2}{|c|}{ Angle " $a$ " } & \multirow{2}{*}{ Ratio } & \multicolumn{2}{|c|}{ Angle " $a$ " } & \multirow{2}{*}{ Ratio } & \multicolumn{2}{|c|}{ Angle " $a$ " } \\
\hline & $e_{1}+/ e_{3}+$ & $e_{1}-/ e_{3}-$ & & $\mathrm{e}_{1}+/ \mathrm{e}_{3}-$ & $e t-1 e s+$ & & $e_{1}+/ e_{3}-$ & $e_{1}-/ e_{3} t$ \\
\hline $\begin{array}{c}0.0 \\
+0.11 \\
0.23 \\
0.37 \\
0.53 \\
0.74 \\
1.00 \\
1.36 \\
1.88 \\
2.73 \\
4.41 \\
9.40 \\
\infty\end{array}$ & $\begin{array}{c}+90^{\circ} \\
85 \\
80 \\
75 \\
70 \\
65 \\
60 \\
55 \\
50 \\
45 \\
40 \\
35 \\
30\end{array}$ & $\begin{array}{l}-90^{\circ} \\
-95 \\
-100 \\
-105 \\
-110 \\
-115 \\
-120 \\
-125 \\
-130 \\
-135 \\
-140 \\
-145 \\
-150\end{array}$ & $\begin{array}{c}0.0 \\
-0.10 \\
-0.19 \\
-0.27 \\
-0.35 \\
-0.42 \\
-0.50 \\
-0.58 \\
-0.65 \\
-0.73 \\
-0.82 \\
-0.90 \\
-1.00\end{array}$ & $\begin{array}{l}-90^{\circ} \\
-85 \\
-80 \\
-75 \\
-70 \\
-65 \\
-60 \\
-55 \\
-50 \\
-45 \\
-40 \\
-35 \\
-30\end{array}$ & $\begin{array}{r}90^{\circ} \\
95 \\
100 \\
105 \\
110 \\
115 \\
120 \\
125 \\
130 \\
135 \\
140 \\
145 \\
150\end{array}$ & $\begin{array}{r}-1.11 \\
-1.23 \\
-1.37 \\
-1.53 \\
-1.74 \\
-2.00 \\
-2.36 \\
-2.88 \\
-3.73 \\
-5.41 \\
-10.40 \\
-\infty\end{array}$ & $\begin{array}{r}-25^{\circ} \\
-20 \\
-15 \\
-10 \\
-5 \\
0 \\
+5 \\
10 \\
15 \\
20 \\
25 \\
30\end{array}$ & $\begin{array}{r}+155^{\circ} \\
160 \\
165 \\
170 \\
175 \\
+180 \\
-175 \\
-170 \\
-165 \\
-160 \\
-155 \\
-150\end{array}$ \\
\hline
\end{tabular}

The manifest value which is represented by $\mathrm{E}$, is determined by the value of any lead and the angle " $a$." To use the value of Lead I, for example, one takes equation (4) above:

$$
\begin{aligned}
& \text { (4) } \mathrm{e}_{1}=\mathrm{E}_{0} \cos a \\
& \text { (11) } \mathrm{E}=\frac{\mathrm{e}_{1}}{\cos a} \\
& \text { (12) } \mathrm{E}=\mathrm{e}_{1} \frac{1}{\cos a}
\end{aligned}
$$

It follows from this last equation (12) that one can determine the manifest value by multiplying the value of Lead I as measured by the reciprocal of the cosine of the angle " $a$." Table 2 gives the values of $\frac{1}{\cos a}$ at intervals of five degrees. with interpolation values for one 
degree. In the higher values the interpolations lead to slight errors. which are inevitable. (For the angle " $\mathrm{a}$ " $=+90$ degrees we cannot use Lead I as the factor $\frac{1}{\cos a}$ is infinity; hence Lead III is substituted.) Suppose, for example, Lead $1=5$ and " $a$ " +65 degrees. Then $\frac{1}{\cos a}=2.37$, which multiplied by 5 gives 11.85 , the value of $E$.

Table 2.-The Value of $\frac{1}{\cos a}$. The Manifest Value Is Obtained by Multiplying the Value of Lead I by This Factor

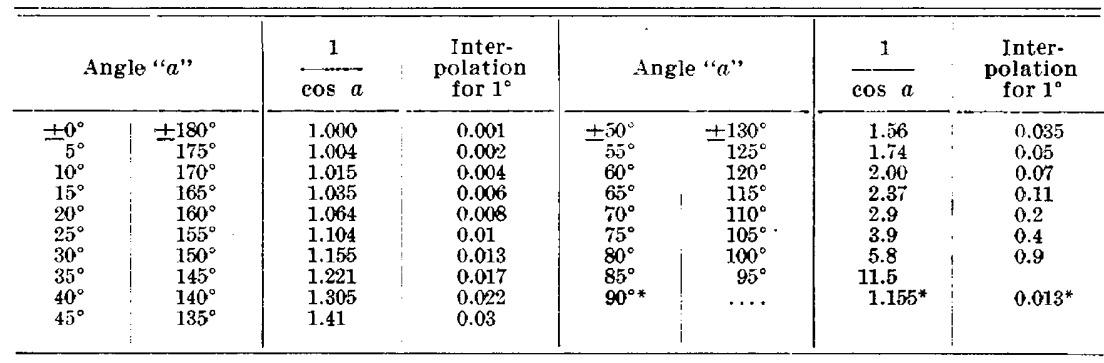

* For " $a "=90^{\circ}$ use lead III.

It should be emphasized that all these "methods" have the same underlying principle and with the exception of that of Waller as explained above, are designed to reach the same result.

In practice two difficulties arise in connection with the determination of the angle " $a$." First, since the electrical axis is constantly shifting throughout the cycle of the heart beat, what axis or axes shall be determined? For certain purposes it is helpful and of great interest to determine the direction of the axis at short intervals throughout the cycle or a part of it. Tabulation of the results, showing clearly the mode of shifting present, is very instructive. ${ }^{14}$ But for practical reasons this is only possible in a limited number of cases. In general, the axis is determined only for that time instant when the greatest potential difference is recorded by one or more of the leads, $i$, e., at the peak of the $Q R$ S complex. This is then spoken of as "the" electrical axis; but in many ways this is misleading. It is not unlikely that as the subject is developed it will appear that the axis should be determined at different instants for different purposes.

Secondly, what parts of the curves in the two or three leads shall we measure? The law of the relationship of the leads is only true of corresponding time instants in the leads. It has been shown that the peaks are often not in phase. Strictly, then, simultaneous leads or leads taken with simultaneous phonocardiograms should be used. ${ }^{15}$ Unfor-

14. Grau, H.: Ueber der Einfluss der Herzlage auf die Form des Flektrokardiogramms, Ztschr. f. klin. Med. 69:281, 1910.

15. Fahr, G.: Simultaneous Records of Heart Sounds and the Electrocardiogram, Heart 4:147 (Nov.) 1912. 
tunately neither of these methods is available for extensive application. Lewis ${ }^{9}$ adjusts enlarged graphs of the three leads, one above the other, until the measured values in all leads at a number of instants are all within the law that Lead II is the sum of Leads I and III. This is the most satisfactory method which is at present available. For the routine determination of the electrical axis in a large number of cases we can only use the values of the peaks of the $Q \mathrm{R} S$ group. In general this gives a value which is not widely at variance with the accurate value of the angle " $a$ " for the time chosen.

TIIE NORMAL ELECTRICAL AXIS

It has been found that the vast majority of normal electrocardiograms show electrical axes at the instant of greatest potential difference that lie within a restricted portion of the compass. Different observers have placed varying limits upon the normal variation but the precise limits are not matters of great importance. Einthoven, Fahr and de Waart $^{8}$ placed the normal limits at 40 and 90 degrees, Waller ${ }^{16}$ at -10 degrees and 100 degrees. Carter and Greene ${ }^{17}$ adopted 0 degrees and 90 degrees which may be followed provided too much emphasis be not placed on slight variations beyond these limits. This limitation certainly embraces nearly all normal cases.

Certain important practical points are explained by consideration of the electrical axis and the manifest value. A few of these may be briefly outlined.

THE EFFECT OF RESPIRATION

The angle " $a$," as is now well-known, changes with respiration, being greater in inspiration and less in expiration. ${ }^{18}$ In more general terms, the same changes occur respectively with descent and ascent of the diaphragm, whatever the cause (compare, for example, the effect of gastric distention). The change in direction of the axis is from 5 to 35 degrees in extent. The form of the electrocardiogram as a rule, is not altered by this change, though the height of the chief ventricular waves in Leads I and III always varies somewhat with the respiratory phase. Lead II shows the variation more rarely and to a less degree for the reason that most axes are more nearly parallel to the plane of that lead and variations in the angle under that condition have less effect upon the value of the lead. This can be seen expressed mathematically for Lead I in Table 2.

16. Waller. A. D.: Various Inclinations of Electrical Axis, Pt. 1A, Proc. Roy. Soc. Lond., B 88:49 (Aug.) 1914.

17. Carter, E. P., and Greene, C. H.: Electrocardiogram and Ventricular Preponderance, Arch. Int. Med. 24:638 (Dec.) 1919.

18. Waller, A. D.: Effect of Respiration on Electrical Axis, J. Physiol. (Proc.) 46:57 (Aug.) 1913. 
It has, however, often been observed ${ }^{19}$ that certain electrocardiograms show a change of $\mathrm{R}$ to $\mathrm{S}$ in Lead III and certain others a change of $\mathrm{S}$ to $\mathrm{R}$ in the same lead. It is Lead III which shows this change because the various axes are usually more nearly at right angles to the plane of this lead and therefore slight changes of direction have marked effects upon the value of the lead. A glance at Figure 1 shows the geometric explanation of the change in direction of the waves. At the angle 30 degrees Lead III is equal to 0 , or, in other words, the current direction is at right angles to the lead plane and no movement of the string is recorded. At angles below 30 degrees Lead III has a negative value and above 30 degrees a positive value. Hence, in a heart whose axis is close to 30 degrees the value of Lead III may be changed from positive to negative by a good expiration; or, vice versa, by a deep inspiration depending on which side of 30 degrees the axis starts from. It should be noted that in most cases these changes are produced only by respiratory movements of more than normal excursion.

The peculiarity of Lead III just discussed underlies the widespread feeling that alterations in this lead should not be emphasized. But it would be better to bear this explanation in mind and use Lead III for whatever information it may contribute. Interestingly enough it will be seen that in cases of "situs inversus" Lead II becomes the unstable lead and Lead III relatively stable.

In measuring curves which show a marked respiratory variation in excursion the high $R$ values in Lead $I$ should be taken in conjunction with low $R$ values or with high $S$ values in Lead III. This follows from the direction in which the axis shifts as explained above.

TILE EFFECTS OF THE POSITION (IF THE BODУ

Moderate changes in the position of the body have but little effect on the electrical axis. The angle " $a$ " is from 10 to 15 degrees greater in the standing than in the sitting or in the prone position. It is usually about the same when the patient is lying on his back as it is when he is seated. In the left lateral decubitus the angle " $a$ " is increased by about 15 degrees, and decreased somewhat less in the right lateral decubitus. ${ }^{10}$

EFFECT OF OTHER DISPLACEMENTS OF THE IIEART

One of the earliest observations:" in this field was of the effect of "situs inversus." Here the effect is complete and the explanation obvious.

19. Waller, A. D.: Voluntary Reversal of Human F.lectrocardiogram by Deep Respiration, J. Physiol. (Proc.) 48:40 (July) 1914. 
Alterations in the position of the heart produced in other ways, in general, do not have marked effects. Pneumothorax, pleural effusion and similar conditions cause but slight shifting of the electrical axis.

VENTRICUL.AR PREPONDERANCE

In the minds of many the subject of the electrical axis is linked inseparably with that of ventricular predominance because it is in this connection that the axis finds its most obvious clinical application. It was first pointed out by Einthoven "that "hypertrophy" of one or the other ventricle is associated with characteristeric electrocardiographic findings, now too well known to need repetition. These peculiarities were definitely related to the anatomical conditions by two series of hearts examined by Lewis ${ }^{20}$ and Cotton. ${ }^{21}$ At the same time, Lewis suggested that in this connection the term "preponderance" rather than "hypertrophy" should be used. It should be recalled that there is normally a left ventricular preponderance present. The normal weight ratio, $\mathrm{L} / \mathrm{R}$, probably ranges between 1.6 and 2.1 . When the expression left preponderance is used, then, it means that the left ventricle is thought of as weighing more than 2.1 times as much as the right ventricle.

The features of the electrocardiogram under discussion are such that they can only be interpreted as reflections of peculiarities of the electrical axis. The "high R" in Lead I and "deep S" in Lead III signify a negative angle " $a$ " ( $\mathrm{S}$ in Lead III first appears at an angle of 30 degrees). The "S" in Lead I and "high R" in Lead III are projections of an axis at an angle greater than 90 degrees ( $S$ in Lead I first appears at that angle). Lewis and his co-workers ${ }^{9}$ have shown that the underlying change in the axis concerns the direction in which it rotates. Normally, the electrical axis rotates uninterruptedly in a clockwise direction. In case of right ventricular preponderance the rotation is in the same direction but very quickly reaches high values for " $a$ " and extends farther. When the left side is preponderant, the direction of rotation is counter-clockwise and the extreme deflections are at negative angles. These departures from the normal are due to a disturbance in the relation between the dextrocardiogram and levocardiogram of which the ordinary electrocardiogram is a summation.

So much it would seem is beyond controversy. Lewis ${ }^{2}$ says there is little reason to doubt that Einthoven's conclusions are valid, and strongly suggests that it should not be assumed in case of incompatibility between clinical and electrocardiographic evidence that the latter

20. Lewis, T.: Observations on Ventricular Preponderance, etc. Heart 5: 367 (July) 1914.

21. Cotton, T.: Observations on Hypertrophy. Heart 6:217 (Oct.) 1917. 
is necessarily at fault. The suggestion that dilatation rather than mass preponderance may sometimes be present has been made by Waller ${ }^{2:}$ and by Fahr, ${ }^{23}$ a suggestion which in the light of our present knowledge needs supporting evidence. In a recent paper Fahr ${ }^{24}$ has advanced the theory that the abnormalities of the electrocardiogram under discussion are due to changes in the relations between the lengths of the bundle branches on the two sides. This theory is at present without a satisfactory experimental basis.

In using the electrical axis for the purpose of estimating ventricular preponderance fixed limits of normality are necessary, and 0 to 90 degrees may be used, provided one bears in mind three points. First, that the normal respiratory variation may be as much as 30 degrees. Secondly, that it is not intended to attach much significance to angles just beyond the limits of normality. And thirdly that there are other causes of abnormal angles, such as "situs inversus" and bundle branch blocks. With regard to the limits defined above, it is easy to remember that the angle " $a$ " is negative when the value of Lead III is negative and the ratio between the value of Lead I and that of Lead III is less than 2 (a negative value in Lead III does not necessarily mean a negative angle). A negative value in Lead $I$ in conjunction with a positive value in Lead III, however, always means an axis at an angle greater than +90 degrees.

Carter and Greene, ${ }^{16}$ in a paper from this laboratory, endeavored to show the general correspondence between the angles of the greatest potential difference developed and the ratios between the weights of the ventricles in the few cases (fifteen in all) in which the necessary data exist. These data are those of Lewis ${ }^{20}$ and Cotton. ${ }^{21}$ It was pointed out that the agreement is, in fact, striking, with the exception of three cases. Explanation of two of these cases was offered by Cotton on the ground that considerable time had elapsed between the taking of the electrocardiogram and the postmortem examination. ${ }^{2.5}$ It was proposed by Carter and Greene to determine the direction of the electrical axis for this purpose by using the values obtained by the algebraic subtraction of the value of $\mathrm{S}$ from that of $\mathrm{R}$ in Leads $\mathrm{I}$ and III on the ground that these waves represent the opposite effects of the two ventricles in the rotation of the axis. The angle " $a$ " thus obtained is to be regarded as a resultant.

22. Waller, A. D.: Electrical Axis of the Human Heart, Lancet 1:1433 (May' 24) ; 1513 (May 31) 1913.

23. Fahr, G.: Clinical Application of Electrocardiography, J. Mich. M. S. 17:10 (Jan.) 1918.

24. Fahr, G. E.: Principles of Electrocardiography, Arch. Int. Med. 27: 126 (Jan.) 1921.

25. Stewart, H. A.: Experimental Contribution to Study of Cardiac Hypertrophy, J. Exper. M. 13:187 (Feb.) 1911. 
This attempt at a quantitive estimation of the electrocardiographic evidence was criticized by Pardee, ${ }^{* 6}$ who concluded that either of two empirical formulas gare the results nearer to the weight ratios. The first of these formulas was used by Lewis in arranging his electrocardiographic findings in the cases in which he had obtained the ventricular weight ratios, at a time when attention had only recently been drawn to the electrical axis. The second is that offered by White and Block. ${ }^{2 i}$ These formulas are essentially similar. Pardee's conclusions were based on the ground that the Lewis and White formulas misplace fewer cases in the weight ratio series. The results are shown graphically in a chart (Fig. 1). This figure is misleading, however, because the weight ratios are represented on one scale, the figures obtained by the formulas on another and the angle " $a$ " on a third scale, so that the results are not comparable.

Examination of Lewis' formula shows that it cannot under all circumstances give a figure representing the electrocardiographic findings. The formula is :

$$
\text { (13) } R_{1}-R_{3}+S_{3}-S_{1}=\text { index. }
$$

This index is 0 when the angle " $a$ " as determined by Carter and Greene is 60 degrees and also when it is -120 degrees. For instance, suppose $\mathrm{R}_{1}=12, \mathrm{~S}_{1}=5, \mathrm{R}_{3}=10, \mathrm{~S}_{6}=3$ (" $a$ " $=60$ degrees) ; and in a second case $\mathrm{R}_{1}=4, \mathrm{~S}_{1}=10, \mathrm{R}_{3}=2, \mathrm{~S}_{3}=8$ (" $a$ ' $=-120$ degrees). In both cases the index is 0 . However one determines the angle " $a$," it is evident that Lewis' figure fails to show the complete reversal of the electrocardiographic data. In general, Lewis' formula gives a high positive figure with left ventricular preponderance and a negative figure with preponderance of the right chamber. A further example of the possible discrepancies may be given from our records:

$\begin{array}{crrrrrr}\text { Case No. } & \mathrm{R}_{1} & \mathrm{~S}_{1} & \mathrm{R}_{1} & \mathrm{~S}_{i} & \text { Lewis' Index } & \text { " } a \text { " } \\ 2314 & 12.0 & 0 . & 4.0 & 1.5 & 9.5 & +39^{\circ} \\ 2237 & 7.0 & 3.0 & 1.5 & 6.5 & 9.0 & -40^{\circ}\end{array}$

Here, again, the electrocardiographic eviclence is certainly not expressed by Lewis' index.

The occurrence of waves of large amplitude in cases of left or right ventricular preponderance is pointed out by Pardee. In the case of left-sided preponderance, the largest waves are all $R_{1}$ or $S_{3}$, while for preponderance of the right ventricle these are $S_{1}$ or $R_{3}$. facts to be expected on the ground of Lewis' explanation of the electrocardiogram as a summation of separate, largely opposing elements due to the spread of the excitation wave through the left and right ventricle

26. Pardee, H. E. B.: Determination of Ventricular Preponderance, Arch. Int. Med. 25:683 (June) 1920.

27. White, P. D., and Bock, A. V.: Electrocardiographic Evidence of Abnormal Ventricular Preponderance and of Auricular Hypertrophy. Am. J. M. Sc. 156:17 (July) 1918 . 
respectively." It seems impossible that consideration of the deflection in one lead alone can be of value, since, for instance, a high $R_{1}$ of given value, has an entirely different significance in conjunction with a negative value in Lead III from that which it has with a positive value in Lead III.

In view of the basis described for the use of the electrical axis in estimating ventricular preponclerance, it does not seem that the method should be discarded because of the discrepancies in the series of fifteen cases referred to above.

\section{AURICLLAR HYPERTROPHY}

There is suggestive evidence that hypertrophy of the auricles is sometimes associated with " $P$ " waves of increased amplitude. ${ }^{2 \pi}$ In estimating the height of the "P" wave the manifest value should be used because of the influence of the direction of the axis on the length of the lead values. In connection with the value of " $\mathrm{P}$ " in Lead I, Table 2 can be used for this purpose.

Space does not permit the analysis, but it can readily be appreciated that an understanding of the principles of the electrical axis is essential to the intelligent interpretation of split and bizarre complexes, and of the curves of premature systoles and bundle branch blocks.

\section{SUMMARY}

1. The definition of the electrical axis is repeated and the various methods proposed for evaluating the angle " $a$ " are outlined.

2. That the angle " $a$ " depends upon the ratio between any two leads is demonstrated and tables for the direct determination of the angle " $a$ " and the manifest value, with a graph for finding the angle by inspection are given.

3. As evidence of the fundamental importance of the electrical axis its relations to the effects of respiration, the position of the body and other causes of displacement of the heart, on the electrocardiogram are pointed out.

4. The use of the angle of the electrical axis at the instant of greatest potential difference in estimating ventricular preponderance is discussed. As far as our knowledge at present goes, this angle probably gives the most accurate indication of the condition present.

Acknowledgment is made of the kind supervision of this paper by Dr. F. P. Carter. 